\title{
CS-NLP team at SemEval-2020 Task 4: Evaluation of State-of-the-art NLP Deep Learning Architectures on Commonsense Reasoning Task
}

\author{
Sirwe Saeedi \\ Aliakbar Panahi \\ Western Michigan University, USA Virginia Commonwealth University, USA \\ sirwe.saeedi@wmich.edu \\ Seyran Saeedi \\ panahia@vcu.edu \\ Alvis C Fong \\ Virginia Commonwealth University, USA Western Michigan University, USA \\ saeedisevcu. edu \\ alvis.fong@wmich.edu
}

\begin{abstract}
In this paper, we investigate a commonsense inference task that unifies natural language understanding and commonsense reasoning. We describe our attempt at SemEval-2020 Task 4 competition: Commonsense Validation and Explanation (ComVE) challenge. We discuss several state-of-the-art deep learning architectures for this challenge. Our system uses prepared labeled textual datasets that were manually curated for three different natural language inference subtasks. The goal of the first subtask is to test whether a model can distinguish between natural language statements that make sense and those that do not make sense. We compare the performance of several language models and fine-tuned classifiers. Then, we propose a method inspired by question/answering tasks to treat a classification problem as a multiple choice question task to boost the performance of our experimental results (96.06\%), which is significantly better than the baseline. For the second subtask, which is to select the reason why a statement does not make sense, we stand within the first six teams (93.7\%) among 27 participants with very competitive results. Our result for last subtask of generating reason against the nonsense statement shows many potentials for future researches as we applied the most powerful generative model of language (GPT-2) with 6.1732 BLEU score among first four teams ${ }^{1}$.
\end{abstract}

Keywords: Artificial Intelligence, Natural Language Processing, Commonsense Reasoning and Knowledge, Language Models, Transformers, Self-Attention.

\section{Introduction}

Commonsense is unstated background knowledge that is used to perceive, infer, and understand the physical world, human emotions, reactions, and knowledge of the common facts that most people agree with. Ordinary commonsense helps us to differentiate between simple false and true statements or answer questions, such as "can an elephant fit into the fridge" quickly, but they can be difficult for automatic systems (Davis, 2017). Recent advances in machine learning emphasize the importance of commonsense reasoning in natural language processing (NLP) and as a critical component of artificial intelligence (AI). In the fifty-year history of AI research, progress was extremely slow (Davis and Morgenstern, 2004) in automated commonsense reasoning. However, when transfer learning (Yosinski et al., 2014; Goodfellow et al., 2016) and then transformers were introduced to the NLP world (Vaswani et al., 2017), great breakthroughs and developments have occurred at an unprecedented pace (Pan and Yang, 2009; Tan et al., 2018). Advances in machine learning and deep learning methods have been achieved in numerous studies and wide range of disciplines (Panahi et al., 2019; Nemati et al., 2020; Sahour et al., 2020; Alshehri et al., 2020; Arodz and Saeedi, 2019; Oh et al., 2018).

This paper describes a system participating in the SemEval-2020 "Commonsense Validation and Explanation (ComVE) Challenge", multiple tasks of commonsense reasoning and Natural Language Understanding (NLU) designed by (Wang et al., 2020). The competition is divided into three subtasks,

\footnotetext{
${ }^{1}$ https://github.com/Sirwe-Saeedi/Commonsense-NLP

This work is licensed under a Creative Commons Attribution 4.0 International Licence. Licence details: http://creativecommons.org/licenses/by/4.0/
} 
which involve testing commonsense reasoning in automatic systems, multiple choice questions, and text generation. In these tasks, participants are asked to improve the performance of previous efforts (Wang et al., 2019a). We apply statistical language modeling, or language modeling (LM) for short as one of the most important parts of modern NLP and then transfer learning to reuse a pretrained model on different data distribution and feature space as the starting point of our target tasks. Applying Transfer Learning to NLP significantly improves the learning process in terms of time and computation through the transfer of knowledge from a related task that has already been learned (Olivas et al., 2009).

Language modeling is the task of probability distribution over sequences of words. It also assigns a probability for the likelihood of a given word (or a sequence of words) to follow a sequence of words. Language modeling are applied to many sorts of tasks, like: Machine Translation, Speech Recognition, Question Answering, Sentiment analysis, etc. AWD-LSTM (ASGD Weight-Dropped LSTM) (Merity et al., 2017) is a fundamental building block of language modeling which uses different gradient update step and it returns the average value of weights in previous iterations instead of current iteration.

Recently, there have been some excellent advancements towards transfer learning, and its success was illuminated by Bidirectional Encoder Representations from Transformers (BERT) (Devlin et al., 2018), OpenAI transformer (GPT-2) (Radford et al., 2019), Universal Language Model Fine-tuning for Text Classification (ULMFiT) by fast.ai founder Jeremy Howard (Howard and Ruder, 2018), ELMo (Peters et al., 2018), and other new waves of cutting-edge methods and architectures like XLNet (Yang et al., 2019), Facebook AI RoBERTa: A Robustly Optimized BERT Pretraining Approach (Liu et al., 2019), ALBERT: A Lite BERT for Self-supervised Learning of Language Representations (Lan et al., 2019), T5 team google (Raffel et al., 2019), and CTRL (Keskar et al., 2019). For this work, we employ and fine-tune some of these suitable models.

When BERT was published, it achieved state-of-the-art performance on a number of natural language understanding tasks. As opposed to directional models like word2vec (Mikolov et al., 2015), which generates a single word representation for each word in the vocabulary and read the text input sequentially, BERT is deeply bidirectional and reads the entire sequence of words at once. Therefore, BERT allows the model to learn the context of a word based on all of its surroundings using two training strategies: Masked Language Model (MLM) and Next Sentence Prediction (NSP). MLM technique is masking out some of the words in the input and then condition each word bidirectionally to predict the masked words. A random sample of the tokens in the input sequence is selected and replaced with the special token '[MASK]' and the objective is a cross-entropy loss on predicting the masked tokens (Devlin et al., 2018). In this paper, we use a method inspired by MLM.

The paper "Attention Is All You Need" (Vaswani et al., 2017) describes a sequence-to-sequence architecture called transformers relying entirely on the self-attention mechanism and does not rely on any recurrent network such as GRU (Chung et al., 2014) and LSTM (Hochreiter and Schmidhuber, 1997). Transformers consist of Encoders and Decoders. The encoder takes the input sequence and then decides which other parts of the sequence are important by attributing different weights to them. Decoder turns the encoded sentence into another sequence corresponding to the target task.

A huge variety of downstream tasks have been devised to test a model's understanding of a specific aspect of language. The General Language Understanding Evaluation (GLUE) (Wang et al., 2018) and The Natural Language Decathlon (decaNLP) benchmarks (McCann et al., 2018) consist of difficult and diverse natural language task datasets. These benchmarks span complex tasks, such as question answering, machine translation, textual entailment, natural language inference, and commonsense pronoun resolution. The majority of state-of-the-art transformers models publish their results for all tasks on the GLUE benchmark. For example, models like different modified versions of BERT, RoBERTa, and T5 outperform the human baseline benchmark (Zhu et al., 2019; Wang et al., 2019b). For the evaluation phase, GLUE follows the same approach as SemEval.

Our attempts at SemEval-2020 Task4 competition boost performance on two subtasks significantly. Our idea in reframing the first subtask helps to outperform results of state-of-the-art architecture and language models like BERT, AlBERT, and ULMFiT. General-Domain of ULMFiT is to predict the next word in a sequence by a widely used pretrained AWD-LSTM network on the WikiText-103 dataset. ULMFiT 
could outperform many text classification tasks like emotion detection, early detection of depression, and medical images analysis (Lundervold and Lundervold, 2019; Xiao, 2019; Trotzek et al., 2018). We were ranked 11 with a very competitive result on the first subtask and achieved rank 6 for the second subtask amongst 40 and 27 teams, respectively.

This paper is organized as follows. In Section 2, we introduce the three subtasks and their datasets. In Section 3, we describe our different applied models and various strategies that were used to fine tune the models for each individual subtask. In Section 4, we present the performance of our system. Finally, we conclude the paper in Section 5.

\section{Task Definition and Datasets}

As discussed, SemEval-2020 Task 4 consists of three subtasks, each designed for a different natural language inference task. Figure 1 shows a sample for each subtask and the corresponding answer of the model.

- SubtaskA (Commonsense Validation): Given two English statements with similar wordings, decide which one does not make sense. We had access to 10,000, and 2021 human-labeled pairs of sentences for training and trial models, respectively. After releasing the dev set, we combined these two datasets for training phase and used the dev set to test our models.

- SubtaskB (Explanation): Given the nonsense statement, select the correct option among three to reason why the statement conflicts with human knowledge. The number of samples in the datasets for this task is similar to subtaskA. Each sample contains the incorrect statement from subtaskA and three candidate reasons to explain why this is against commonsense knowledge.

- SubtaskC (Reason Generating): Given the nonsense statement, generate an understandable reason in the form of a sequence of words to verify why the statement is against human knowledge. Training samples of datasets for this subtask are all of the false sentences in subtaskA as well as for trial and dev set.

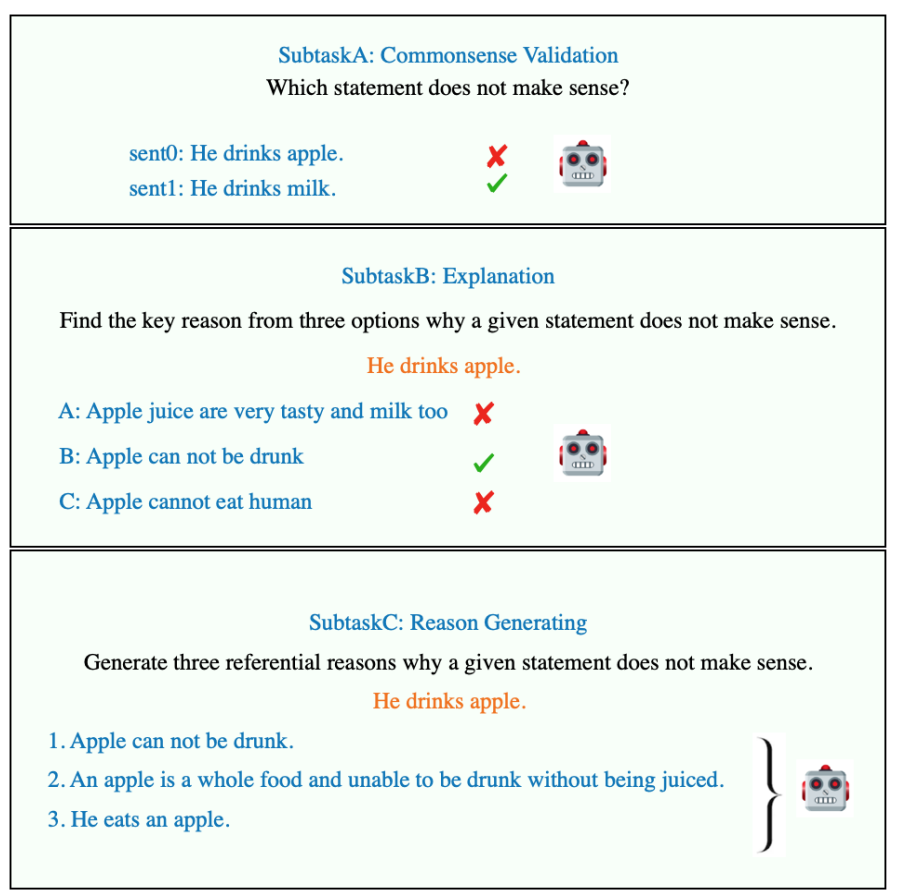

Figure 1: Sample of training data for each subtask 


\section{Model Description}

Large pretrained language models are definitely the main trend of the latest NLP breakthroughs. As transformers occupy the NLP leaderboards, we choose several state-of-the-art architectures to outperform the baseline of all subtasks significantly. For each subtask, we describe our system separately below.

\subsection{SubtaskA (Commonsense Validation)}

We consider two approaches to address this task: the first method is based on language models, and the second approach uses classifiers. Our experimental process begins with language models which the key idea behind the first approach is to find the probability of appearing each word in statements and then select one with higher multiplication of probabilities.

Our first try involves fine-tuning pretrained model on AWD-LSTM (as described in Section 1), which performs as poor as a random guess. We also try two other different language models: 'BERT' the MLM that attempts to predict the original value of the masked words, based on the non-masked words in the sequence of words, and then transformer.

Original BERT uniformly selects $15 \%$ of the input tokens for possible replacement. Of the selected tokens, $80 \%$ are replaced with '[MASK]', $10 \%$ are left unchanged, and $10 \%$ are replaced by a randomly selected vocabulary token. However, our way of using MLM follows these steps:

1. Add special tokens to the beginning and end of each sentence.

['[CLS]', 'He', 'drinks', 'apple', '[SEP]']

2. Replace each token from left to right by '[MASK]' each time.

$$
\begin{aligned}
& \text { ['[MASK]', 'He', 'drinks', 'apple', '[SEP]'], } \\
& \text { ['CCLS]', '[MASK]', 'drinks', 'apple', '[SEP]'], } \\
& \text { ['[CLS]', 'He', '[MASK]', 'apple', '[SEP]'], } \\
& \text { ['[CLS]', 'He', 'drinks', '[MASK]', '[SEP]'], } \\
& \text { ['[CLS]', 'He', 'drinks', 'apple', '[MASK]'] }
\end{aligned}
$$

3. Feed them to MLM for predicting the probabilities of the original masked tokens.

4. Normalize predicted probabilities using softmax activation function in the output layer.

5. Multiply predicted probabilities of masked tokens for each pair of statement. The correct sentence has a higher probability.

During the consideration of dataset homogeneity, we observe some samples are ended by periods and some others are not. The most frequent reason for using periods is to mark the end of sentences that are not questions or exclamations. By adding a period at the end of all statements, we increase the accuracy by $4 \%$, which is remarkable. We also try normalization and padding to boost performance of the model to minimize the impact of sequence length. Surprisingly, during normalization in step 4 we observe that normalizing by the length of sequence root of multiplied probabilities does not improve the performance of model. Similarly, normalization using perplexity to evaluate language models does not increase the accuracy of model. Perplexity is the inverse probability of the test set, normalized by the number of words and minimizing perplexity is the same as maximizing probability. We observe that padding does not make any differences in terms of accuracy. Therefore, the result of BERT MLM is almost the same with the baseline, which is achieved by fine-tuned ELMo as reported by (Wang et al., 2019a). As a result, our observation shows BERT MLM model is more suitable for long document understanding; however, the maximum length (27) of our samples is too short.

As mentioned, we consider classifiers as the second approach to deal with this task. We show that the classification-based approach is more efficient in recognizing nonsense statements except ULMFiT for text classification. Our main reasons for applying ULMFiT to address subtaskA are its techniques to deal with a small domain of dataset: Discriminative fine-tuning, slanted triangular learning rates instead of 
using the same learning rate throughout training, and gradual unfreezing neural network layers. However, applying ULMFiT for this task is similar to choosing between any two statements, randomly. On the other hand, our results show the fine-tuned classifier on the pretrained AWD-LSTM, transformer, and random guess yielded results with almost close to 50\% accuracy. As shown in Table 1, these models can not differentiate sentences that make sense from those that do not make sense, properly.

In addition, we apply the ubiquitous architecture of transformers for classification, such as BERT, Albert, and RoBERTa. All these models allow us to pretrain a model on a large corpus of data, such as all Wikipedia articles and English book corpus, and then fine-tune them on downstream tasks. Looking at Table 1, we see RoBERTa outperforms all other models. We find out a significant difference when using fine-tuned Albert and BERT classification. Table 1 summarizes the performance of these systems on dev in terms of accuracy.

\begin{tabular}{|l|l|}
\hline Models & Accuracy \\
\hline AWD-LSTM & 52.45 \\
\hline Transformer & 53.8 \\
\hline ULMFiT & 59.8 \\
\hline BERT MLM & 74.29 \\
\hline BERT classification & 88 \\
\hline Albert classification & 92 \\
\hline RoBERTa classification & 95 \\
\hline RoBERTa multiple choice question & 96.08 \\
\hline
\end{tabular}

Table 1: Experimental results for subtaskA on dev set.

Our idea to boost the performance of all these applied models is reframing the input of subtaskA as a binary classification task to the input of another downstream task, multiple choice questions. As a result, we show fine-tuned RoBERTa for multiple choice questions task gives better results than RoBERTa for classification problem on both dev and test set (See Table 1).

The difference between these two models is paying attention to the statements. In the self-attention layer, the encoder looks at other words in the input sentence as it encodes a specific word. For binary classification models like BERT, RoBERTa, and Albert, we concatenate two statements and then selfattention layer attends to each position in the input sequence, including both statements. However, for RoBERTa multiple choice questions task, we feed each statement to the network separately. Therefore, the attention layer attends to the sequence of words for each individual statement for gathering information that can lead to better encoding for each word.

Question answering task usually provides a paragraph of context and a question. The goal is to answer the question based on the information in the context. For subtaskA, we do not have the context and question; all we have is two options corresponding to the statements which are fed to the network, separately. Our goal is to select the correct statement (answer) from the two options.

As expected, determining optimal hyper-parameters has a significant impact on the accuracy on the performance of the model, and their optimization needs careful evaluation of many key hyper-parameters. We primarily follow the default hyper-parameters of RoBERTa, except for the maximum sequence length, weight decay, and learning rate $\in\{1 e-5,2 e-5,3 e-5\}$ which is warmed up over 320 steps with a maximum of 5336 numbers of step to a peak value and then linearly decayed. The other hyper-parameters remained as defaults during the training process for 5 epochs. By searching the hyperparameter space for the optimum values, fine-tuned hyper-parameters achieve $96.08 \%$ and $94.7 \%$ accuracy on dev and test set, respectively. Our result is a big jump from $74.1 \%$ baseline accuracy and competes with $99.1 \%$ accuracy of human performance.

\subsection{SubtaskB (Explanation)}

As described earlier, subtaskB requires world knowledge and targets commonsense reasoning to answer why nonsense statements do not make sense. This type of task seems trivial for humans with a basic 
knowledge but is still one of the most challenging tasks in the NLP world. However, the baseline for human performance, $97.8 \%$ shows how it is difficult to reason even with a comprehensive commonsense knowledge.

Our goal is to investigate whether transformers like RoBERTa (which its performance was confirmed on subtaskA) can learn commonsense inference given a nonsense statement. The architecture of RoBERTalarge is comprised of 24-layer, 1024-hidden dimension, 16-self attention heads, 355M parameters and pretrained on book corpus plus English Wikipedia, English CommonCrawl News, and WebText corpus.

SubtaskB is a multiple choice question task and we fine-tune hyper-parameters of RoBERTa model to answer questions. In this setting, we concatenate the nonsense statement (context) with each option (endings) and then use three statements as the input of model. For example, 'He drinks apple.' is the context and ['Apple juice are very tasty and milk too.', 'Apple can not be drunk.', 'Apple cannot eat a human.'] is the list of endings. We want to select the ending from three options that is entailed by the context:

- "He drinks apple. Apple juice are very tasty and milk too."

- "He drinks apple. Apple can not be drunk."

- "He drinks apple. Apple cannot eat a human."

The set of concatenated examples is fed into the model to predict the answer of questions that require reasoning. We considered a few hyper-parameter settings and figured out the model with hyper-parameters in Table 2 yields the surprising results $93.7 \%$, compared to the baseline accuracy of $45.6 \%$.

\begin{tabular}{|l|l|}
\hline hyper-parameters & value \\
\hline batch size & 16 \\
\hline learning rate & $1 e-5$ \\
\hline weight decay & 0.1 \\
\hline adam epsilon & $1 e-8$ \\
\hline num_train_epochs & 5 \\
\hline max_steps & 5336 \\
\hline warmup_steps & 320 \\
\hline
\end{tabular}

Table 2: Tuned hyper-parameters of RoBERTa for subtaskB.

\subsection{SubtaskC (Reason Generating)}

Based on the subtaskC definition, we can frame subtaskC as a conditional text generation problem. Given a nonsense statement, we expect that the language model will generate commonsense reasons to explain why statement conflicts with our knowledge. We applied the full version of OpenAI GPT2 (Generative Pre-Training), a large-scale unsupervised language model with billions of parameters, trained on a very large corpus of text data. The goal of this model is to automatically generate text, given a sequence of natural language words. The performance of GPT-2 in a zero-shot setting is competitive on many language modeling datasets and various tasks like reading comprehension, translation, and question answering.

GPT-2 architecture claims that the model performs well in generating coherent samples depending on the context, which are fairly represented during the training process. However, we observed that employing GPT-2 for generating texts against the given nonsense statements is poor in performance with unnatural topic switching and 6.1732 BLEU score. We used the Pytorch implementation of GPT-2 (with all default hyper-parameters) that is provided by Huggingface transformers (Wolf et al., 2019) for natural language generation.

The GPT-2 is built using transformer decoder blocks. The key behined GPT-2 is called "auto-regression" that outputs one token at a time and after each token is produced, that token is added to the sequence of inputs then the new produced sequence becomes the input to the model. 
Notably, we submitted the original test set (including nonesense statements) for the evaluation phase on SemEval-2020 portal and surprisingly, we stand among the first four teams. The competitive BLEU score of 17.2 with the top team shows that subtaskC is challenging enough to receive more research attentions. We believe that our simple and naive efforts indicate significant opportunities for future research to utilize reasoning on commonsense knowledge.

\section{Conclusion}

We evaluated architectures for three commonsense reasoning tasks. First, we found that RoBERTa-large performs better substantially in differentiating sentences that make sense from those that do not make sense compared to other cutting-edge architectures (e.g. Albert, BERT, and ULMFiT). We reframe this classification task to a question answering task to enhance the performance of the fine-tuned RoBERTa to $96.08 \%$. Second, we achieved significant results on reasoning why false statements do not make sense. We showed that RoBERTa performs well in selecting the correct option among three to infer the commonsense reason and it yields significant result with $93.7 \%$ accuracy compare to baseline using BERT, $45.6 \%$. With a little effort on generating reasons to explain why false statement conflicts with commonsense knowledge, we observe that the original test set produces 17.2 BLEU score which ranked us among first four teams in the competition with a very competitive results. Our experimental result showed that GPT-2 performs as poor as random generating of a sequence of words for this task. We believe this task has many potentials and challenges for upcoming NLP researches. As another future work, we believe that ensemble learning can reduce the variance of predictions and also improve prediction performance. In ensemble learning, multiple models are generated and combined to address the subtasks and reduce the likelihood of an unfortunate selection of a poor one.

\section{References}

Fahad Alshehri, Mohamed Sultan, Sita Karki, Essam Alwagdani, Saleh Alsefry, Hassan Alharbi, Hossein Sahour, and Neil Sturchio. 2020. Mapping the distribution of shallow groundwater occurrences using remote sensingbased statistical modeling over southwest saudi arabia. Remote Sensing, 12.

Tomasz Arodz and Seyran Saeedi. 2019. Quantum sparse support vector machines. arXiv preprint arXiv:1902.01879.

Junyoung Chung, Caglar Gulcehre, KyungHyun Cho, and Yoshua Bengio. 2014. Empirical evaluation of gated recurrent neural networks on sequence modeling. arXiv preprint arXiv:1412.3555.

Ernest Davis and Leora Morgenstern. 2004. Introduction: Progress in formal commonsense reasoning. Artificial Intelligence, 153(1-2):1-12, March. Logical Formalizations and Commonsense Reasoning ; Conference date: 01-05-2001 Through 01-05-2001.

Ernest Davis. 2017. Logical formalizations of commonsense reasoning: A survey. Journal of Artificial Intelligence Research, 59:651-723, May.

Jacob Devlin, Ming-Wei Chang, Kenton Lee, and Kristina Toutanova. 2018. Bert: Pre-training of deep bidirectional transformers for language understanding. arXiv preprint arXiv:1810.04805.

Ian Goodfellow, Yoshua Bengio, and Aaron Courville. 2016. Deep Learning. MIT Press. http://www . deeplearningbook.org.

Sepp Hochreiter and Jürgen Schmidhuber. 1997. Long short-term memory. Neural computation, 9(8):1735-1780.

Jeremy Howard and Sebastian Ruder. 2018. Universal language model fine-tuning for text classification. arXiv preprint arXiv:1801.06146.

Nitish Shirish Keskar, Bryan McCann, Lav R Varshney, Caiming Xiong, and Richard Socher. 2019. Ctrl: A conditional transformer language model for controllable generation. arXiv preprint arXiv:1909.05858.

Zhenzhong Lan, Mingda Chen, Sebastian Goodman, Kevin Gimpel, Piyush Sharma, and Radu Soricut. 2019. Albert: A lite bert for self-supervised learning of language representations. arXiv preprint arXiv:1909.11942. 
Yinhan Liu, Myle Ott, Naman Goyal, Jingfei Du, Mandar Joshi, Danqi Chen, Omer Levy, Mike Lewis, Luke Zettlemoyer, and Veselin Stoyanov. 2019. Roberta: A robustly optimized bert pretraining approach. arXiv preprint arXiv:1907.11692.

Alexander Selvikvåg Lundervold and Arvid Lundervold. 2019. An overview of deep learning in medical imaging focusing on mri. Zeitschrift für Medizinische Physik, 29(2):102-127.

Bryan McCann, Nitish Shirish Keskar, Caiming Xiong, and Richard Socher. 2018. The natural language decathlon: Multitask learning as question answering. arXiv preprint arXiv:1806.08730.

Stephen Merity, Nitish Shirish Keskar, and Richard Socher. 2017. Regularizing and optimizing lstm language models. arXiv preprint arXiv:1708.02182.

Tomas Mikolov, Kai Chen, Gregory S Corrado, and Jeffrey A Dean. 2015. Computing numeric representations of words in a high-dimensional space, May 19. US Patent 9,037,464.

Mohammadreza Nemati, Jamal Ansary, and Nazafarin Nemati. 2020. Machine learning approaches in covid-19 survival analysis and discharge time likelihood prediction using clinical data. Patterns, page 100074.

Jun-Seok Oh, Valerian Kwigizile, Ron Van Houten, Ahmad Feizi, Majid Mastali, et al. 2018. Effects of safe bicycle passing laws on drivers' behavior and bicyclists' safety. Technical report, Western Michigan University.

Emilio Soria Olivas, Jose David Martin Guerrero, Marcelino Martinez Sober, Jose Rafael Magdalena Benedito, and Antonio Jose Serrano Lopez. 2009. Handbook Of Research On Machine Learning Applications and Trends: Algorithms, Methods and Techniques - 2 Volumes. Information Science Reference - Imprint of: IGI Publishing, Hershey, PA.

Sinno Jialin Pan and Qiang Yang. 2009. A survey on transfer learning. IEEE Transactions on knowledge and data engineering, 22(10):1345-1359.

Aliakbar Panahi, Seyran Saeedi, and Tom J Arodz. 2019. word2ket: Space-efficient word embeddings inspired by quantum entanglement. ArXiv, abs/1911.04975.

Matthew E. Peters, Mark Neumann, Mohit Iyyer, Matt Gardner, Christopher Clark, Kenton Lee, and Luke Zettlemoyer. 2018. Deep contextualized word representations. In Proc. of NAACL.

Alec Radford, Jeffrey Wu, Rewon Child, David Luan, Dario Amodei, and Ilya Sutskever. 2019. Language models are unsupervised multitask learners. OpenAI Blog, 1(8):9.

Colin Raffel, Noam Shazeer, Adam Roberts, Katherine Lee, Sharan Narang, Michael Matena, Yanqi Zhou, Wei Li, and Peter J Liu. 2019. Exploring the limits of transfer learning with a unified text-to-text transformer. $a r X i v$ preprint arXiv:1910.10683.

Hossein Sahour, Mohamed Sultan, Mehdi Vazifedan, Karem Abdelmohsen, Sita Karki, John Yellich, Esayas Gebremichael, Fahad Alshehri, and Tamer Elbayoumi. 2020. Statistical applications to downscale grace- derived terrestrial water storage data and to fill temporal gaps. Remote Sensing, 12:533, 02.

Chuanqi Tan, Fuchun Sun, Tao Kong, Wenchang Zhang, Chao Yang, and Chunfang Liu. 2018. A survey on deep transfer learning. In ICANN.

Marcel Trotzek, Sven Koitka, and Christoph M Friedrich. 2018. Utilizing neural networks and linguistic metadata for early detection of depression indications in text sequences. arXiv preprint arXiv:1804.07000.

A Vaswani, N Shazeer, N Parmar, J Uszkoreit, L Jones, AN Gomez, L Kaiser, and I Polosukhin. 2017. Attention is all you need. arxiv 2017. arXiv preprint arXiv:1706.03762.

Alex Wang, Amanpreet Singh, Julian Michael, Felix Hill, Omer Levy, and Samuel R Bowman. 2018. Glue: A multi-task benchmark and analysis platform for natural language understanding. arXiv preprint arXiv:1804.07461.

Cunxiang Wang, Shuailong Liang, Yue Zhang, Xiaonan Li, and Tian Gao. 2019a. Does it make sense? and why? a pilot study for sense making and explanation. arXiv preprint arXiv:1906.00363.

Wei Wang, Bin Bi, Ming Yan, Chen Wu, Zuyi Bao, Liwei Peng, and Luo Si. 2019b. Structbert: Incorporating language structures into pre-training for deep language understanding. arXiv preprint arXiv:1908.04577. 
Cunxiang Wang, Shuailong Liang, Yili Jin, Yilong Wang, Xiaodan Zhu, and Yue Zhang. 2020. SemEval-2020 task 4: Commonsense validation and explanation. In Proceedings of The 14th International Workshop on Semantic Evaluation. Association for Computational Linguistics.

Thomas Wolf, Lysandre Debut, Victor Sanh, Julien Chaumond, Clement Delangue, Anthony Moi, Pierric Cistac, Tim Rault, Rémi Louf, Morgan Funtowicz, et al. 2019. Huggingface's transformers: State-of-the-art natural language processing. ArXiv, pages arXiv-1910.

Joan Xiao. 2019. Figure eight at semeval-2019 task 3: Ensemble of transfer learning methods for contextual emotion detection. In Proceedings of the 13th International Workshop on Semantic Evaluation, pages 220-224.

Zhilin Yang, Zihang Dai, Yiming Yang, Jaime Carbonell, Russ R Salakhutdinov, and Quoc V Le. 2019. Xlnet: Generalized autoregressive pretraining for language understanding. In Advances in neural information processing systems, pages 5754-5764.

Jason Yosinski, Jeff Clune, Yoshua Bengio, and Hod Lipson. 2014. How transferable are features in deep neural networks? In NIPS.

Chen Zhu, Yu Cheng, Zhe Gan, Siqi Sun, Tom Goldstein, and Jingjing Liu. 2019. Freelb: Enhanced adversarial training for natural language understanding. In International Conference on Learning Representations. 